\title{
Scaffold to selectivity
}

Oncogenic mutations in the BRAF gene (such as V600E) correlate with increased severity and decreased response to chemotherapy in several human tumours. Inhibition of oncogenic B-Raf kinase activity could therefore be of therapeutic benefit, and now Tsai and colleagues report the discovery of an orally available, selective B-Raf ${ }^{\mathrm{V} 600 \mathrm{E}}$ kinase inhibitor with potent antimelanoma activity.

The authors used a scaffold- and structure-based approach to identify inhibitors. An initial kinase-inhibition screen of 20,000 low molecular mass 'scaffolds' against divergent kinases was used to identify promising compounds for co-crystallization with target kinases, and 100 compound-bound kinase structures were solved.

From this, a scaffold candidate was chosen for the optimization of potency and selectivity. Screening of compounds predicted to have the most productive binding interactions revealed a series containing a difluorophenylsulphonamide substructural motif that displayed potency and selectivity for oncogenic B-Raf. These compounds were then co-crystallized with engineered forms of B-Raf ${ }^{\mathrm{V} 600 \mathrm{E}}$ and wild-type B-Raf. Based on these co-crystal structures, subsequent optimization chemistry enabled the identification of the lead compound PLX4720.
To understand the high affinity and selectivity of PLX4720, the authors determined the crystal structure of the B-Raf-PLX4720 complex. The crucial determinant for oncogenic selectivity is derived from the interaction between the sulphonamide and the beginning of the DFG region - three amino acids essential for coordination of the ATP substrate - of the kinase. This interaction subsequently directs the attendant alkyl chain into a small pocket unique to the Raf family. A selectivity mechanism was determined, whereby a deprotonated sulphonamide favours an interaction with the active kinase conformation that is also favoured by the V600E mutation.

When PLX4720 was tested on a panel of melanoma cell lines, the compound had antimelanoma activity - measured by cell-cycle arrest and apoptotic induction - in B-Raf ${ }^{\mathrm{V} 600 \mathrm{E}}$-positive cells. PLX4720 was then shown to inhibit the growth of melanoma-based synthetic skin derived from B-Raf ${ }^{\mathrm{V} 600 \mathrm{E}}$ malignant cells that are inherently resistant to pharmacological intervention.

Last, in a mouse tumour xenograft model (bearing the $B R A F^{\mathrm{V} 600 \mathrm{E}}$ mutation), once-daily oral treatment with PLX4720 resulted in a substantial block of tumour growth, and in some cases tumours were completely

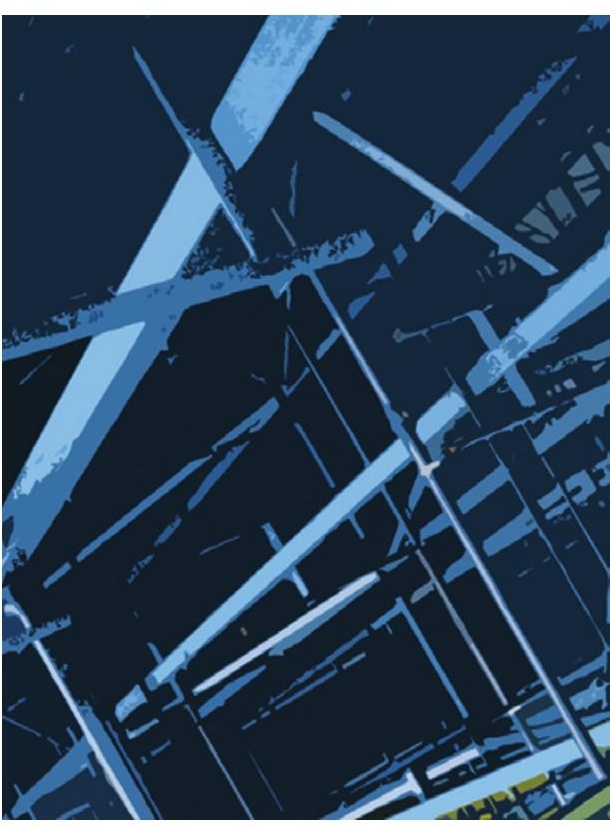

destroyed. In this model, PLX4720 was well tolerated with no adverse effects.

This study provides rationale for the use of PLX4720 as a treatment for patients with advanced-stage melanoma. Furthermore, crystallography studies showed that the selective targeting of oncogenic B-Raf mutants can be achieved by small-molecule inhibitors, and this should aid the development of future therapeutic kinase inhibitors for the treatment of melanoma and other cancers.

Charlotte Harrison

ORIGINAL RESEARCH PAPER Tsai, J. et al. Discovery of a selective inhibitor of oncogenic B-Raf kinase with potent antimelanoma activity. Proc. Natl Acad. Sci. USA 105, 3041-3046 